\title{
Disentangling neutrino-nucleon cross section and high energy neutrino flux with a $\mathrm{km}^{3}$ neutrino telescope
}

\author{
E. Borriello ${ }^{1,2}$, A. Cuoco ${ }^{3}$, G. Mangano ${ }^{1}$, G. Miele ${ }^{1,2}$, S. Pastor ${ }^{2}$, O. Pisanti ${ }^{1}$, and P. D. Serpico ${ }^{4}$ \\ ${ }^{1}$ Università "Federico II" Dipartimento di Scienze Fisiche, 8 INFN Sezione di Napoli, Napoli, Italy \\ 2 AHEP Group, Institut de Física Corpuscular, CSIC/Universitat de València, Apt. 22085, 46071 València, Spain \\ ${ }^{3}$ Department of Physics and Astronomy, University of Aarhus, Ny Munkegade, 8000 Aarhus, Denmark, and \\ ${ }^{4}$ Center for Particle Astrophysics, Fermi National Accelerator Laboratory, Batavia, IL 60510-0500 USA
}

(Dated: November 4, 2018)

\begin{abstract}
The energy-zenith angular event distribution in a neutrino telescope provides a unique tool to determine at the same time the neutrino-nucleon cross section at extreme kinematical regions, and the high energy neutrino flux. By using a simple parametrization for fluxes and cross sections, we present a sensitivity analysis for the case of a $\mathrm{km}^{3}$ neutrino telescope. In particular, we consider the specific case of an under-water Mediterranean telescope placed at the NEMO site, although most of our results also apply to an under-ice detector such as IceCube. We determine the sensitivity to departures from standard values of the cross sections above $1 \mathrm{PeV}$ which can be probed independently from an a-priori knowledge of the normalization and energy dependence of the flux. We also stress that the capability to tag downgoing neutrino showers in the PeV range against the cosmic ray induced background of penetrating muons appears to be a crucial requirement to derive meaningful constraints on the cross section.
\end{abstract}

PACS numbers: 13.15.+g, 95.55.Vj, 95.85.Ry

\section{INTRODUCTION}

High energy neutrino astronomy is one of the most promising research lines in astroparticle physics. Neutrinos are in fact one of the main components of the cosmic radiation in the high energy regime, and although their fluxes are uncertain and depend on the specific production process, their detection would provide valuable information concerning the sources and the acceleration mechanism in extreme astrophysical environments. Similarly to photons and unlike cosmic rays, they keep directional information which can be used to perform astronomy. Differently from gamma rays, they are emitted only in hadronic processes and travel unimpeded through cosmological distances well above the TeV energy. Moreover, due to the peculiar charge assignment of neutrinos in the electroweak standard model, they may be the particles most sensitive to exotic physics. An intriguing possibility is that a measurement of their cross section on ordinary matter in extreme and still unexplored kinematical regions might unveil new physics (see e.g. [1]).

From the experimental point of view, after the first generation of telescopes has proved the viability of the Cerenkov detection technique under deep water (Baikal [2]) and ice (AMANDA [3]) by detecting atmospheric neutrinos, one is probably on the verge of the first detections at the IceCube [4] telescope, being completed at the South Pole, and possibly at the smaller ANTARES [5] telescope under construction in the Mediterranean. Additionally, ANTARES as well as NESTOR [6] and NEMO [7] are involved in R\&D projects aimed at the construction of a km ${ }^{3}$ Neutrino Telescope (NT) in the deep water of the Mediterranean sea, coordinated in the European network KM3NeT [8].

In the present paper we address in a more quantitative way the question of how well a $\mathrm{km}^{3} \mathrm{NT}$ performs in the simultaneous determination of the neutrino flux and the neutrino-nucleon cross section by using as observable the energy-zenith angular event distribution. Note that, unless the spectrum is known a priori, for a NT surrounded by an isotropic medium the cross section and the flux would be completely degenerate. However, under the sole assumption that the incoming spectrum is isotropic, the different opacity of the Earth in different directions - which becomes relevant above $\sim 100 \mathrm{TeV}$ - allows one to disentangle the two observables, as it has been discussed before e.g. in [9, 10, 11]. In other words, a NT is only sensitive to the product of cross section and flux, but the incoming isotropic flux is shielded (via a non-trivial function of the cross section) in a direction-dependent way, which makes possible to break the degeneracy by means of directional information. Here we study this property using a parametric and phenomenological approach, rather than illustrating it with a few discrete cases from models proposed in the literature.

In particular, we consider the specific case of an under-water Mediterranean telescope, although most of our results also apply to an under-ice detector such as IceCube. Since the three different proposed sites for the under-water $\mathrm{km}^{3}$ telescopes have shown event rate differences of the order of $20 \%$ [12], for the sake of brevity we report the results of our analysis for the NEMO site only, which shows intermediate performances.

This paper is structured as follows. In Sec. Пwe introduce the parameterization used for the flux and cross section. In Sec. III we describe the formalism used, while our results are reported in Sec. IV Finally, we conclude in Sec. V] 


\section{PARAMETERIZATION OF FLUX AND CROSS SECTIONS}

Many astrophysical objects are expected to produce a flux of high energy neutrinos, possibly with energies higher than the highest energy cosmic rays ever detected, of the order of $10^{11} \mathrm{GeV}$. The center of mass energy available in a neutrino-nucleon collision for a neutrino with laboratory energy $E_{\nu}$ is $\sqrt{s} \simeq \sqrt{2 E_{\nu} m_{N}} \simeq 1.4 \mathrm{TeV} \sqrt{E_{\nu} / \mathrm{PeV}}, m_{N}$ being the nucleon mass. Already at $\mathrm{PeV}$ incident energies, this is well above the electro-weak symmetry scale of $\mathcal{O}(100) \mathrm{GeV}$ and may thus reveal the onset of physics beyond the standard model ${ }^{1}$. Since neutrinos only respond to weak interactions, even weak effects may be revealed by a study of the neutrino-nucleon cross sections at NTs. One example is provided by low-scale quantum gravity models, see e.g. [14].

Unfortunately, there are two major problems to be addressed in such a program: the low statistics expected at NTs, which requires huge volumes and long operation times, and the ignorance of the diffuse astrophysical spectrum. At the range of energy $E_{\nu} \sim \mathrm{PeV}$ which starts to be interesting for particle physics constraints, the natural target of NTs are diffuse fluxes rather than point-like sources. On the basis of existing predictions for extragalactic fluxes, we limit our analysis to energies above $10^{-0.5} \mathrm{PeV}$, where isotropic diffuse extragalactic fluxes are expected to dominate over the steeper atmospheric neutrino flux. Around PeV energies, the atmospheric neutrino event rates are very small even in a $\mathrm{km}^{3}$ telescope and the only remaining background is given by penetrating muons from extensive showers due to cosmic ray events, which may require an active veto to be disentangled from the signal. Given the observed behavior of cosmic ray and gamma ray spectra, it is reasonable to parameterize the neutrino spectra as a power law or, more generally, as a broken power-law. The latter parameterization would be probably needed when considering the flux over several decades of energy. However, for the following discussion most of the useful events will have primary energy $E_{\nu}<10 \mathrm{PeV}$, and the single power-law approximation seems reasonable for a single decade in energy. The spectral index in Fermi-like acceleration mechanisms is of the order of -2 or slightly steeper, but this observable is expected to be determined by the data as well. In the following, we parameterize the neutrino flux (per flavor, summing neutrinos and anti-neutrinos) as

$$
\phi_{\nu}\left(E_{\nu}\right) \equiv \frac{\mathrm{d}^{2} \Phi_{\nu}}{\mathrm{d} E_{\nu} \mathrm{d} \Omega}\left(E_{\nu}\right)=1.3 \cdot 10^{-20} C\left(\frac{E_{\nu}}{1 \mathrm{PeV}}\right)^{-2 D} \mathrm{GeV}^{-1} \mathrm{~cm}^{-2} \mathrm{~s}^{-1} \mathrm{sr}^{-1},
$$

where $C$ and $D$ are normalization and flux steepness free parameters, and for $C=D=1$ one recovers the benchmark case of a Waxman-Bahcall flux [1, 15].

Concerning the cross sections, we adopt a simple parameterization as a broken power-law where each energy interval (fixed a priori) has its own slope, and continuity is imposed at the boundaries. For example, the charged current (CC) cross section is given by

$$
\frac{\sigma_{C C}^{\nu N}}{10^{-33} \mathrm{~cm}^{2}}=\left\{\begin{array}{ll}
0.344\left(\frac{E_{\nu}}{E_{1}}\right)^{0.492 A} & E_{1} \leq E_{\nu} \leq E_{2} \\
0.344\left(\frac{E_{2}}{E_{1}}\right)^{0.492 A}\left(\frac{E_{\nu}}{E_{2}}\right)^{0.492 B} & E_{\nu}>E_{2}
\end{array},\right.
$$

where we assume $E_{1}=10^{-0.5} \mathrm{PeV}, E_{2}=1 \mathrm{PeV}$ and, for $A=1$, the expression for the first energy bin reduces to the standard value reported in [16]. An analogous parameterization is used for the neutral current (NC) cross section,

$$
\frac{\sigma_{N C}^{\nu N}}{0.418 \cdot 10^{-33} \mathrm{~cm}^{2}}=\left\{\begin{array}{ll}
0.344\left(\frac{E_{\nu}}{E_{1}}\right)^{0.492 A^{\prime}} & E_{1} \leq E_{\nu} \leq E_{2} \\
0.344\left(\frac{E_{2}}{E_{1}}\right)^{0.492 A^{\prime}}\left(\frac{E_{\nu}}{E_{2}}\right)^{0.492 B^{\prime}} & E_{\nu}>E_{2}
\end{array} .\right.
$$

In the following, we shall consider both cases where $\mathrm{CC}$ and $\mathrm{NC}$ cross sections change proportionally to each other (i.e., $A=A^{\prime}$ and $\left.B=B^{\prime}\right)$ and the case where only $\sigma_{\mathrm{NC}}$ is affected $\left(A=B=1\right.$ while $A^{\prime}$ and $B^{\prime}$ are set free). The former possibility mimics the scenario, for example, where the current extrapolation of parton distribution functions is wrong. It is more likely, though, that "truly exotic" new physics would manifest itself only in NC events; the latter case is a toy model for this class of situations. There are also cases where new physics manifests additionally in changes of the inelasticity of the cross sections, but we leave them outside the range of possibilities explored in this analysis: so, we shall make standard assumptions on the inelasticity of the collisions, as reported in more detail in the next Section.

\footnotetext{
${ }^{1}$ Of course, instruments looking at the highest energy events, say above $10^{10} \mathrm{GeV}$, have greater chances to detect significant departures from the standard model behavior, although the limited statistics likely requires future space based fluorescence detector (for an analysis of this case, see for example [13]).
} 


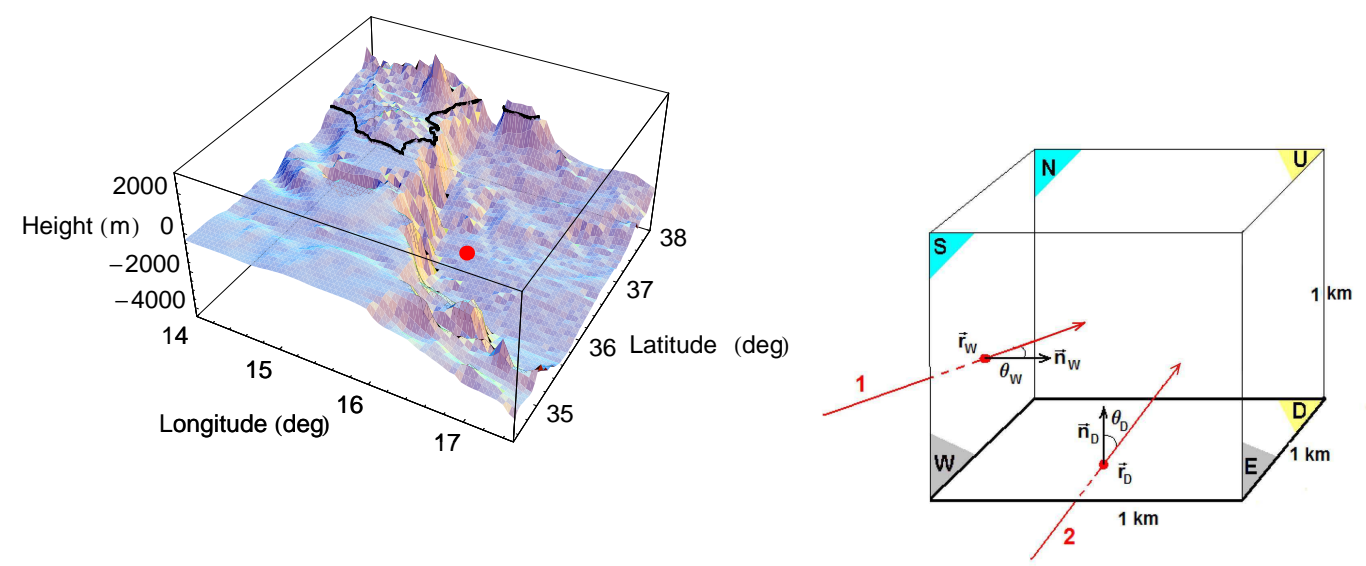

FIG. 1: On the left side is reported the surface profile of the area nearby the NEMO site, with the red spot giving the NT location. On the right, we show the assumed NT fiducial volume and the related notations (see also Ref. [12]).

\section{THE FORMALISM}

To perform our forecast, we follow the formalism outlined in [12. We generate neutrino tracks isotropically outside the Earth and assume flavor equipartition due to neutrino oscillations and pion production dominance [17]. We place then a $\mathrm{km}^{3}$ NT at the NEMO site, see left panel of Fig. 1 For simplicity we assume the telescope fiducial volume as a cube bounded by six lateral surfaces, labeled D, U, S, N, W, and E for the Down, Up, South, North, West, and East side, respectively (see right panel of Fig. 1).

Despite the different behavior of the produced tau leptons with respect to muons in terms of energy loss and decay length, both $\nu_{\mu}$ and $\nu_{\tau}$ event detection rates are sensitive to the matter distribution in the neighborhood of the NT site. In principle, the elevation profile of the Earth surface around the telescope may be relevant, similarly to what is known for Earth-skimming ultra-high energy $\nu_{\tau}$ 's at extensive air shower detectors, also investigated by some of the present authors [18] for the Pierre Auger Observatory [19] by using a Digital Elevation Map (DEM) of the site. In Ref. [12] the DEM's of the under-water Earth surface provided by the Global Relief Data survey (ETOPO2) 20] were used to estimate the effective aperture for $\nu_{\tau}$ and $\nu_{\mu}$ detection of a $\mathrm{km}^{3} \mathrm{NT}$ in the Mediterranean sea placed at any of the three locations proposed by the ANTARES, NEMO and NESTOR collaborations. It was found that the effect of the profile on the total number of events is negligible, although it may be important in the differential number of events from different directions, provided one has an excellent angular resolution. In the present analysis we take into account the underwater surface profile as done in Ref. [12], although for the present results it plays a minor role. Moreover we also take into account the radial density profile of the Earth (reported, for example, in Ref. [21]).

Neutrino telescopes were originally thought mostly as $\nu_{\mu}$ detectors, but they have some sensitivity to all neutrino flavors [22], although this does not trivially translate into a flavor tagging sensitivity. In particular, their capability as $\nu_{\tau}$ detectors has become a hot topic in view of the fact that flavor neutrino oscillations lead to nearly equal astrophysical fluxes for the three neutrino flavors. While electron neutrinos only induce shower events inside the fiducial volume, muon and very high energy tau leptons produced in charged current neutrino interactions create tracks which may be detected even when originating far from the instrumented volume. In the following, we consider as experimental observables:

(i) The energy deposited in the detector, $\Delta E$.

(ii) The topology of the event, namely if it is a shower or a track event.

(iii) Some (at least loose) information on the incoming direction of the event.

In turn, these observables depend on some detector-dependent parameters like the optical properties of the medium, the geometry of the strings and the position of the optical modules on it, the efficiencies, etc. Here we take the simplified approach to consider a (muon or tau) track detected whenever the charged lepton decay length is longer than the length of the intersection of the trajectory with the instrumented cubic volume. Any other neutral current event which forms inside the instrumented volume, or charged current event which does not fulfill the previous 

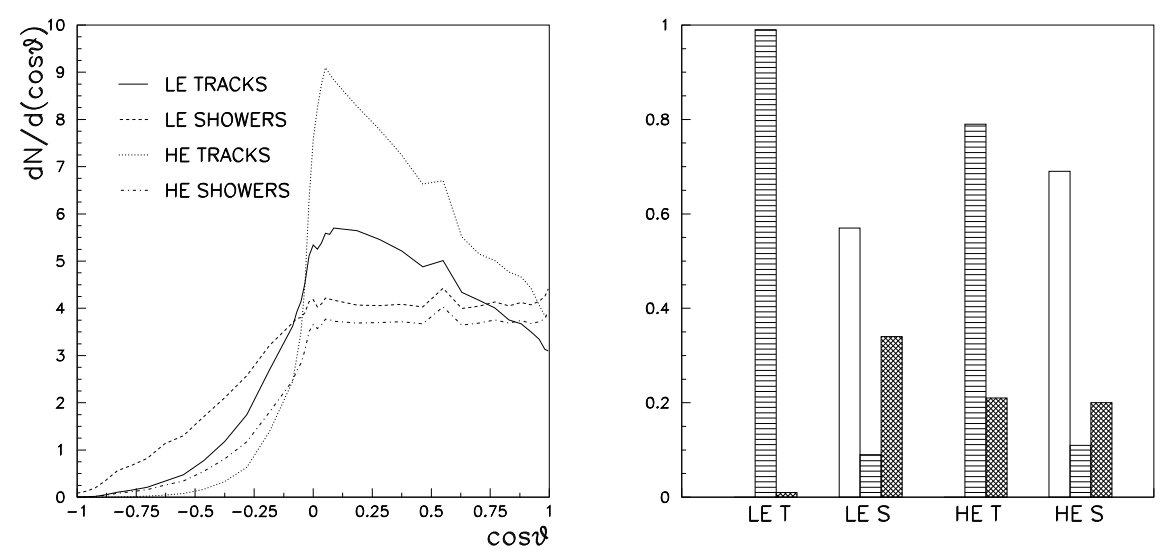

FIG. 2: In the left panel we give the zenith angle distribution of events of different kind and at different energy for the fiducial case $A=B=C=D=1$ and in one year of collection time. In the right panel we report the fractional contribution of the three flavors (empty, horizontal, and full hatching for electrons, muons, and taus, respectively) to the different type of events ( $\mathrm{T}$ stands for tracks, $\mathrm{S}$ for showers), assuming flavor equipartition in the incoming fluxes.

condition is classified as a shower. Although the actual criteria in a realistic experiment will differ, we expect this simplification to catch the physics of the problem. We shall comment on the impact of dropping the assumption of topological discrimination in Sec. IV]

Concerning the energy deposited in the detector, for our purposes it is sufficient to consider two bins only: a low-energy one (denoted LE) corresponding to $10^{-0.5} \leq \Delta E / \mathrm{PeV} \leq 1$ and a high-energy one (denoted HE) for $\Delta E / \mathrm{PeV}>1$. Note that we choose the lower bounds of the two bins equal to the quantities $E_{1}$ and $E_{2}$ in Eq.s (2) and (3). Of course, this is a conventional choice dictated by simplicity and others are possible, although a posteriori it reveals to be a quite reasonable one. In order to calculate the energy released in the detector, one must also know the fraction of invisible energy and the energy loss mechanism of charged leptons. The fraction of energy not carried by the outgoing lepton and thus released to the struck nucleon is the inelasticity, $y$. At these energies $y$ is basically independent of the flavor and is the same for $\nu$ and $\bar{\nu}$, decreasing from $\sim 0.3$ at $E_{\nu} \simeq E_{1}$ to $\sim 0.2$ at the highest energies of interest [21]. If the interaction happens inside the detector (contained event) the energy $y E_{\nu}$ is considered observed, since most of the energy in the hadronic shower generated by the struck hadron is released locally, virtually appearing as a point-like source ${ }^{2}$. For $\mathrm{NC}$ events, this is the only energy release, since the outgoing neutrino is invisible. For CC events, all of the remaining energy $(1-y) E_{\nu}$ is released locally for electron contained or other shower events, while for muon or tau tracks the energy losses are calculated as in [24, 25]. Namely, the differential energy loss of the $\tau$ leptons per unit of length in an underwater NT can be simply taken as $\mathrm{d} E_{\tau} / \mathrm{d} \lambda=-\beta_{\tau} E_{\tau} \varrho_{w}$, with $\beta_{\tau}=0.71 \times 10^{-6} \mathrm{~cm}^{2} \mathrm{~g}^{-1}$ and $\varrho_{w}$ denoting the water density. Analogously, for muons one just needs to replace $\beta_{\tau}$ with the corresponding value, $\beta_{\mu}=0.58 \times 10^{-5} \mathrm{~cm}^{2} \mathrm{~g}^{-1}$.

We end up with four different categories of events: LE tracks, LE showers, HE tracks, and HE showers. In the left panel of Fig. 2 we illustrate the zenith angle distribution of the events of different kind and at different energy for the fiducial case $A=B=A^{\prime}=B^{\prime}=C=D=1$ and in one year of collection time. First note that, in the case of an equal cross section in the two bins, the corresponding LE and HE curves should have the same shape, differing only in the normalization. The fact that this is not the case is a manifestation of the separate sensitivity of NTs to the cross section and the flux. The angular shapes of these curves are plausible, too. For downgoing events $(\cos \vartheta>0)$ the interaction probability of neutrinos is $\ll 1$. Tracks can propagate into the detector even when forming relatively far from it; thus, the larger the grammage crossed the more numerous the events. The most notable manifestation of this effect is given by the peak near the horizon $(\cos \vartheta \simeq 0)$ due to the so called Earth-skimming events. The distribution of downgoing showers is instead flat. This is because contained events are the dominant contribution to this class, and the previous enhancement factor due to leptons created far away is not present. Instead, the distribution of the upgoing events $(\cos \vartheta<0)$ is more and more suppressed towards $\cos \vartheta=-1$. This is due to the fact that at

\footnotetext{
${ }^{2}$ Even the highest energy showers penetrate water or ice less than $\sim 10 \mathrm{~m}$, a distance short compared to any reasonable spacing of the photomultiplier tubes (PMTs). The radius over which PMT signals are produced is $\sim 250 \mathrm{~m}$ for a $1 \mathrm{PeV}$ shower; this radius grows or decreases by approximately $50 \mathrm{~m}$ with every decade of shower energy [23].
} 
these energies the Earth is opaque to neutrinos, an effect most pronounced for events closer to the nadir. For tracks the suppression is even more pronounced, since it involves not only the suppression of the neutrino flux entering the detector, but also the additional energy loss for charged lepton tracks forming outside the instrumented volume. Comparing LE and HE events, note that HE events suffer a higher opacity, so the upgoing rate suppression is more pronounced with respect to the LE case. On the other hand, the range of charged leptons is larger, which explains the steeper behavior of the HE downgoing tracks curve. The number of downgoing track events also grows at HE, since the cross section is higher and we are in a regime where the interaction probability is $\ll 1$. The spiky bumps at $\cos \vartheta \simeq \pm 0.6$ are simply an effect of the cubic geometry assumed (there is more instrumented volume when looking at angles closer to a vertex of the cube). In the right panel of Fig. 2. we show the flavor composition of the four classes of events. As expected, basically only muons contribute to the LE track bin. Indeed, the tau decay length is $D_{\tau} \simeq 50 \mathrm{~m}\left(E_{\tau} / \mathrm{PeV}\right)$. A realistic cut due to the spacing of the towers would probably remove even this small tau contamination. On the other side, the tau contribution to shower events decreases with energy, due to the increase of the relativistic tau gamma factor. Although we shall not discuss this point further here, it has been noted that the fact that different types of events are not flavor blind offers another possibility for astrophysical diagnostics 26$]$.

Although the overall number of events at a NT is limited, the previous discussion suggests that at very least a further partition of each of the previous bins in upgoing or downgoing events would greatly improve the diagnostic power for disentangling cross section from flux. Note that it is indeed a very weak requirement for a NT to be able to reconstruct at least the sign of the cosine of the zenith angle, in order to reject the background from downgoing muons from cosmic ray showers. Even so, it is at present unclear how well one can tag downgoing events as a signal against the cosmic-ray background. Around $\mathrm{PeV}$ energies, this should be feasible in IceCube, where the IceTop surface array offers a veto for cosmic ray events. For a Mediterranean $\mathrm{km}^{3}$ neutrino telescope no final design exists, but the possibility has been suggested to extend the detection capability of the experiment with some sea top stations [27]. Keeping this possibility in mind, we shall consider both the case in which downgoing signal events can be used and the one where this is not possible. Also, we have tested that slightly changing the angular intervals and/or adding a third angular bin have only a minor effect on the sensitivity in the parameter space.

For each choice of parameters, we end up with the counts $N_{i, \alpha}^{K}$, where $K$ labels the type/topology of the event, $i$ the energy bin and $\alpha$ the angular one. As already mentioned, we always consider two energy bins: a low-energy one, corresponding to $E_{1} \leq \Delta E \leq E_{2}$ and a high-energy one, corresponding to $\Delta E>E_{2}$. Regarding the choice of the angular binning, when both the downgoing and upgoing information is used, we divide the angular range in the two bins $\left[0^{\circ}, 90^{\circ}\right]$ and $\left[90^{\circ}, 180^{\circ}\right]$ while, if only upgoing events are included in the analysis, we consider the two bins $\left[90^{\circ}, 107^{\circ}\right]$ and $\left[107^{\circ}, 180^{\circ}\right]$. We fix $C=1$ since it is just a normalization, simply correlated to the exposure time needed to achieve the proper event statistics. The remaining parameters vary in a grid of $125+125$ theoretical models as follows: $D \in[0.5,1.5]$ in steps of 0.25 , while $A=A^{\prime}, B=B^{\prime} \in[0,4]$ in steps of 1 or $A=B=1, A^{\prime}, B^{\prime} \in[0,4]$ in steps of 1 in the two cases under investigation, that is: 1) CC and NC cross sections vary proportionally to each other; 2) only $\mathrm{NC}$ cross section changes. To determine approximately the range of parameters, we require that at least one event falls in each bin in one year of running. We then compare the counts $N_{i, \alpha}^{K}$ with the expected counts $C_{i, \alpha}^{K}$ for the benchmark case $A=B=A^{\prime}=B^{\prime}=C=D=1$ by means of a multi-Poisson likelihood analysis [28], in which the likelihood function, $\mathcal{L} \propto \exp \left(-\chi^{2} / 2\right)$, is defined using the following expression for the $\chi^{2}$ :

$$
\chi^{2}=2 \sum_{i, \alpha, K}\left[N_{i, \alpha}^{K}-C_{i, \alpha}^{K}+C_{i, \alpha}^{K} \ln \left(\frac{C_{i, \alpha}^{K}}{N_{i, \alpha}^{K}}\right)\right] .
$$

\section{RESULTS}

In this Section we present the results of our analysis. Fig.s 3 and 4 are obtained considering the case where CC and NC cross sections change proportionally to each other. The solid (black) curves in the panels of Fig. 3 show the marginalized constraints on couples of parameters (contours at $68 \%$ and $95 \%$ CL) in the case one has both upgoing and downgoing information available, for five years of exposure. Dashed (red) contours represent the case where no topological information is assumed, i.e. when one sums track and shower events. In general, there is a negative correlation between the cross section parameters $A$ and $B$, since to some extent a higher cross section in the range $E_{1}<E<E_{2}$ can be compensated by a smaller one in the higher energy range (note also that in the second line of Eq. (2) both $A$ and $B$ enter). Indeed, the primary energies are not directly observable, while the energy losses $\Delta E$ are. Also, there are negative correlations between $A$ and $C$ or $D$. The reason is that a higher cross section bin can be somewhat compensated by a smaller flux normalization $(C)$ or a slightly steeper flux spectrum $(D)$. The anti-correlation between $A$ and $B$ explains the correlations between $B$ and $C$ and $B$ and $D$. In all cases the contours close, and the parameters can be determined to some accuracy. The degeneracy between flux and cross section is thus 

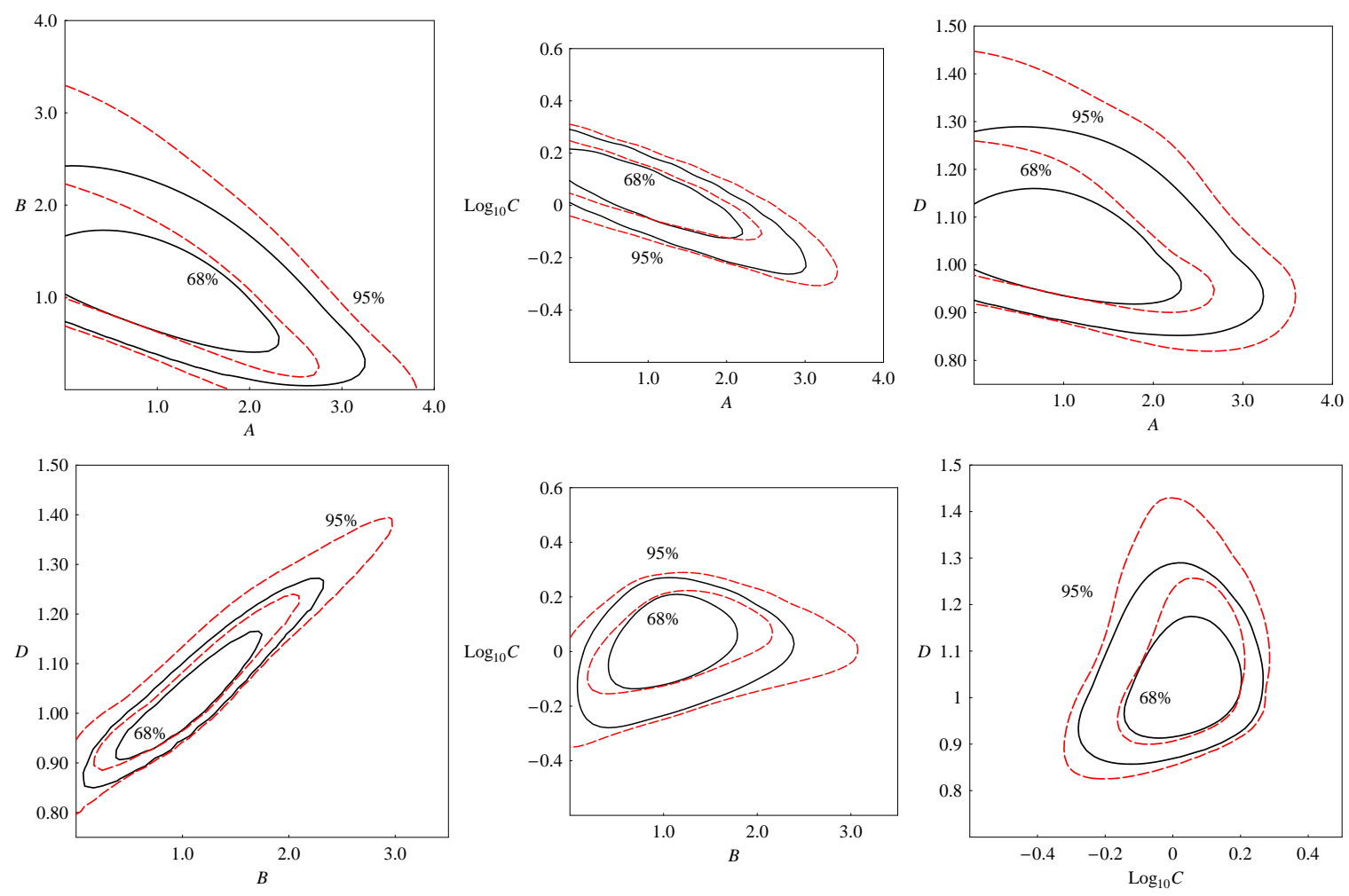

FIG. 3: For the case where CC and NC cross sections change proportionally to each other, the solid (black) contours (68\% and $95 \% \mathrm{CL}$ ) represent marginalized constraints on pairs of parameters $\{A, B, C, D\}$ in the case where both upgoing and downgoing neutrino events can be tagged, for five years of exposure. Dashed (red) contours represent the case where no topological information is assumed, i.e. when one sums track and shower events.

broken by the combined use of angular and energy independent information. Note how the topological information adds diagnostic power, although does not change the qualitative behavior.

In Fig. 4 we show instead the marginalized constraints on couples of parameters (contours at $68 \%$ and $95 \%$ CL) in the case where only upgoing events can be tagged, again for five years of exposure. Solid (black) curves correspond to the case of two bins, $\left[90^{\circ}, 107^{\circ}\right]$ and $\left[107^{\circ}, 180^{\circ}\right.$, while dashed (red) curves show the results when these two bins are merged together. Only the flux parameters can be determined to some accuracy, even if some sensitivity to $A$ and $B$ is still recovered in the case of two angular bins. The basic physical reason for this behavior has been discussed analytically in [10]. As long as $\mathrm{NC}$ and $\mathrm{CC}$ cross sections change proportionally, then both the upward track rate and the upward shower rate are independent of cross sections, with or without new physics involved. If the energy

\begin{tabular}{ccc}
\hline \hline & $68 \% \mathrm{CL}$ & $95 \% \mathrm{CL}$ \\
\hline \hline$A$ & $1.0_{-0.7}^{+0.6}$ & $1.0_{-1.0}^{+1.2}$ \\
\hline$B$ & $1.2_{-0.5}^{+0.4}$ & $1.2_{-0.8}^{+0.7}$ \\
\hline $\log _{10} C$ & $0.04 \pm 0.10$ & $0.04_{-0.19}^{+0.15}$ \\
\hline$D$ & $1.0 \pm 0.1$ & $1.0_{-0.1}^{+0.2}$ \\
\hline \hline
\end{tabular}

\begin{tabular}{ccc}
\hline \hline & $68 \% \mathrm{CL}$ & $95 \% \mathrm{CL}$ \\
\hline \hline$A^{\prime}$ & $<2.0$ & $<3.4$ \\
\hline$B^{\prime}$ & $<1.4$ & $<2.4$ \\
\hline $\log _{10} C$ & $-0.01_{-0.13}^{+0.11}$ & $-0.01_{-0.24}^{+0.20}$ \\
\hline$D$ & $1.1 \pm 0.1$ & $1.1 \pm 0.2$ \\
\hline \hline
\end{tabular}

TABLE I: Determination of cross section and flux parameters, obtained by marginalizing the (black) solid contours of Fig. 3 (left table) and of Fig. 5 (right table). 

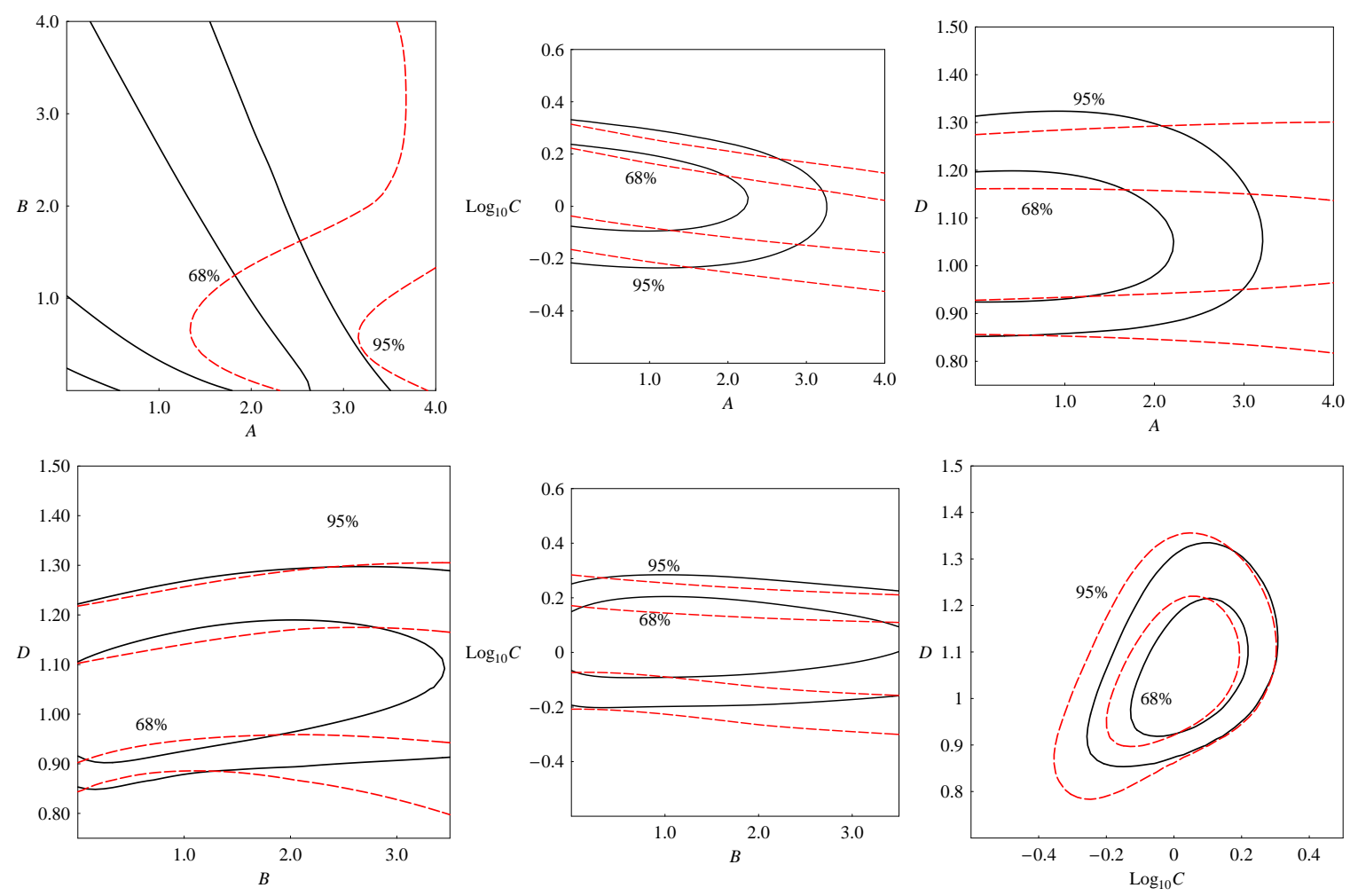

FIG. 4: For the case where CC and NC cross section change proportionally to each other, we report the marginalized constraints on couples of parameters $\{A, B, C, D\}$ (contours at $68 \%$ and $95 \%$ CL) in the case where only upgoing neutrino events can be tagged and split in two angular bins, for five years of exposure. Track vs. shower separation capability is assumed. Dashed (red) contours represent the previous case where all events are summed in a single angular bin.

dependence of the cross sections involved is the same, as we assume here for the fiducial case, the two observables (upgoing track and shower events) are just proportional to the integrated flux in the corresponding energy bin.

The point we want to emphasize here is that without the tagging capability of downgoing events, there is little hope for NTs to get meaningful constraints on the cross sections. A further binning of the upgoing events into two angular bins may only help a little bit, with the additional problem that the statistics is more limited in each bin.

Finally, the curves in Fig.s 5 and 6 correspond to the solid (black) lines in Fig.s 3 and 4 but assuming that only $\mathrm{NC}$ are changed (for illustrative purposes, we fix here $A=B=1$ for CC). The flux parameters $C$ and $D$ are quite accurately determined, in particular $D$ is fixed at $10 \%$ level, since the CC cross section contributing to tracks and, partially, to shower events is assumed to be known in this case. There is instead quite a poor determination of the $\mathrm{NC}$ cross section parameters, and in particular of $A^{\prime}$. Indeed, only values of $A^{\prime} \geq 3.4$ and $B^{\prime} \geq 2.4$ are ruled out at $95 \% \mathrm{CL}$. This result is easy to understand. In fact, the largest contribution to NC shower events corresponds to downgoing neutrinos; so the event rate is almost independent of the intervening matter in the neighborhood of the NT site, being simply proportional to the product of the flux times cross section. For example, at high energies the number of NC events increases more than logarithmically as function of the highest possible neutrino energy: for $B^{\prime}>D / 0.492 \sim 2$ it becomes unacceptably large and thus excluded (actually the result would diverge if $B^{\prime} \geq 2$ in the unphysical case of an incoming flux with $D=1$ for arbitrary high energies.) The other relevant feature is the sensitivity only to a combination of $A^{\prime}$ and $B^{\prime}$ (roughly $A^{\prime}+\kappa B^{\prime}$, with $\kappa \simeq 1.5$ for the case of Fig. 5). The reason is that, due to the smaller energy transferred in visible channels in $\mathrm{NC}$ events, the $\mathrm{NC}$ events collected in both our energy bins are mostly sensitive to the high energy behavior of the cross section, i.e. the second line in Eq. (3). This function depends on a quasi-degenerate combination of the two parameters, explaining the results. Of course, a larger statistics at higher energies would eventually break this degeneracy, but would probably require too long exposure times.

In Table凹we summarize our results by reporting the parameter sensitivity at $68 \%$ and $95 \%$ after the marginalization of the likelihood function for both scenarios of CC and NC cross sections changing proportionally (left table) and only NC varying and standard behavior of CC (right table). The results are shown only for the best cases with both upgoing and downgoing tagged events and with track vs. shower events discrimination. 

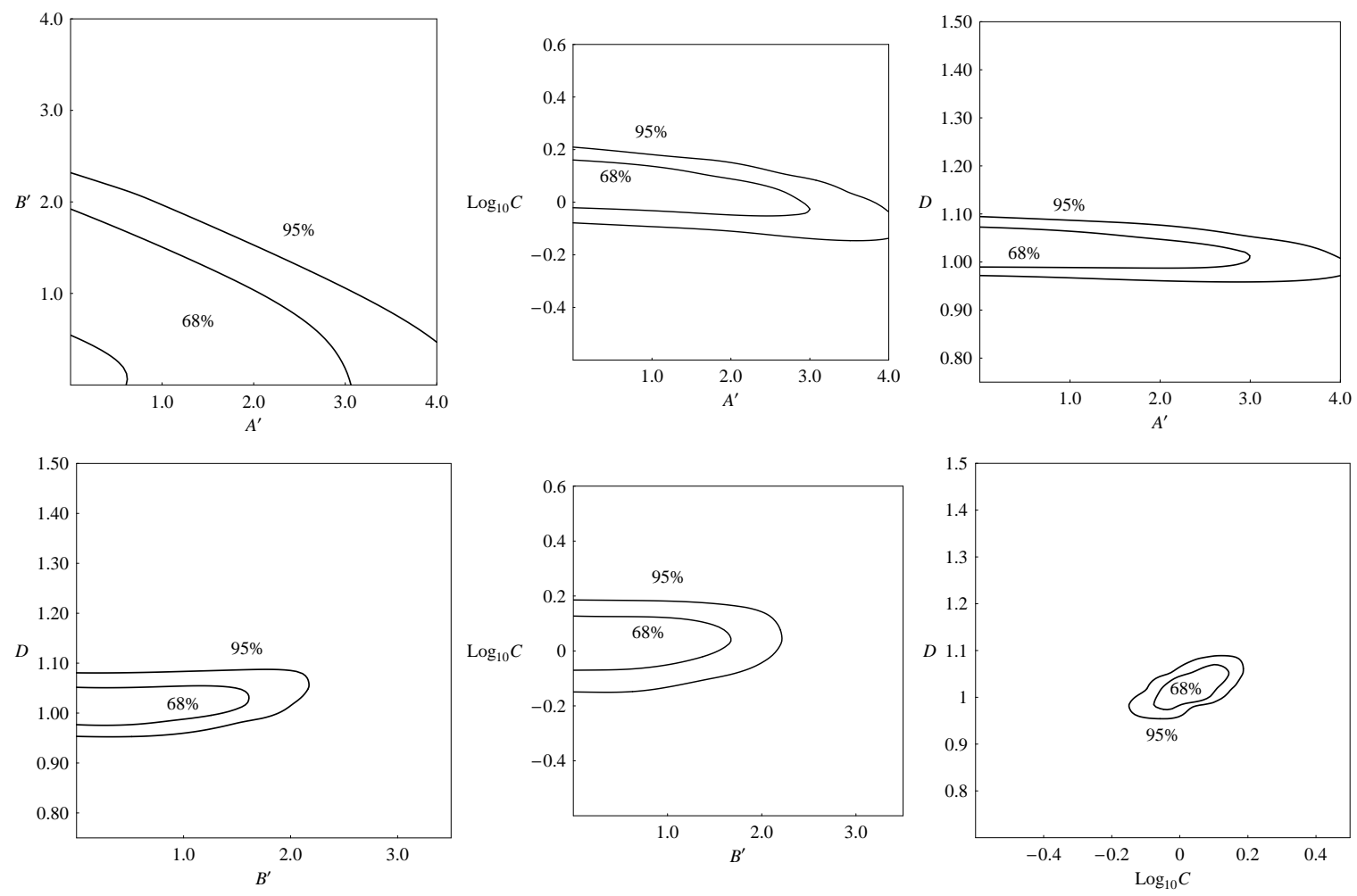

FIG. 5: For the case where only NC cross section changes while $\mathrm{CC}$ one assumes its standard value, we show the marginalized constraints on couples of parameters $\left\{A^{\prime}, B^{\prime}, C, D\right\}$ (contours at $68 \%$ and $95 \% \mathrm{CL}$ ) in the case where both upgoing and downgoing neutrino events can be tagged, for five years of exposure.
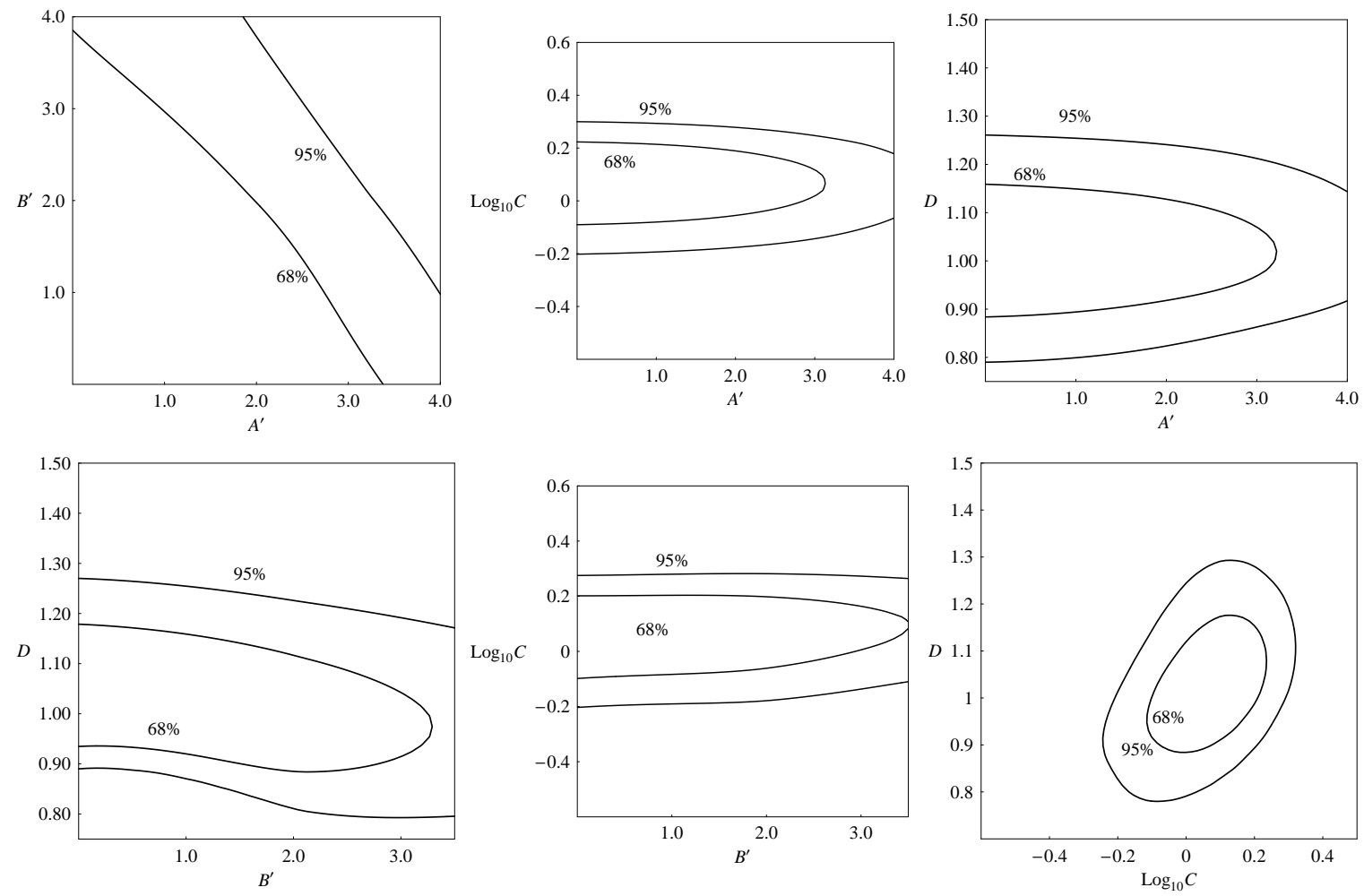

FIG. 6: Same of Fig. 5 but in the case where only upgoing neutrino events can be tagged and split in two angular bins, for five years of exposure. Track vs. shower separation capability is assumed. 


\section{DISCUSSION AND CONCLUSIONS}

In this paper we have performed an analysis of the capability of a $\mathrm{km}^{3} \mathrm{NT}$ to disentangle the high energy diffuse neutrino flux and the neutrino-nucleon cross section in the PeV energy region for the incoming neutrinos. As well known, a separation is possible by exploiting the energy-zenith angular event distribution. Differently from previous treatments, we have used a simple phenomenological parametrization for fluxes and cross sections, presenting a sensitivity analysis in a given parameter space, rather than for a few benchmark cases. Our forecast is thus independent from strong assumptions on the normalization or energy dependence of the flux. The results we obtain are summarized in Fig.s 2 3 4, 5, 6, and refer both to the cases where CC and NC change proportionally to each other, or the case where only NC events are affected by new physics.

One important conclusion is that the capability to tag downgoing neutrino showers in the PeV energy range against the cosmic ray induced background of penetrating muons appears to be a crucial requirement to derive meaningful constraints on the cross section. In this case energy and zenith angle information on diffuse neutrino flux event rate in a NT (upgoing vs. downgoing) would greatly improve the diagnostic power for disentangling cross section from flux.

Of course, our analysis presents several approximations. In particular, while we took into account the geometry of the site (for illustration we considered the NEMO site in the Mediterranean), we did not consider specific information on the detector geometry, efficiency, etc. Also, we assumed that in muon and tau tracks the energy loss is deterministic, rather than stochastic. A more accurate account of the telescope and of the energy losses is beyond the scope of this work. Yet, we expect that it would confirm our qualitative findings, while possibly worsening the accuracy with which parameters can be realistically reconstructed. Since our forecasts are likely more optimistic than a realistic treatment would show, our conclusion on the importance of downgoing event tagging is reinforced. In general, the background due to single energetic muons generated from cosmic rays and surviving deep underground drops faster with energy than the expected neutrino signal, and well above the PeV region the contamination should be negligible. However, detailed simulation are required to settle down this issue quantitatively; an active veto may help pushing down in energy the region where the background can be disentangled from the signal on an event-by-event basis. For the IceCube telescope, in the PeV energy range downgoing muons from cosmic ray showers should be vetoed thanks to the IceTop array. For the Mediterranean $\mathrm{km}^{3}$ neutrino telescopes no final design is available, yet. Given the importance of the present topic, we believe this is an important issue to be addressed in the design phase of these telescopes.

Even in the case where no downgoing tagging for neutrinos will be available for a Mediterranean NT, still its upgoing events at the $\mathrm{PeV}$ would provide important constraints on the flux and cross section if a combined analysis of its data with IceCube ones is performed. At very least, it should allow to test the hypothesis that the diffuse neutrino fluxes at the $\mathrm{PeV}$ from the two hemispheres are consistent with each other, thus validating the hypothesis of an overall isotropic flux.

\section{Acknowledgments}

P.D. Serpico acknowledges support by the US Department of Energy and by NASA grant NAG5-10842. G. Miele acknowledges supports by Generalitat Valenciana (ref. AINV/2007/080 CSIC) and by PRIN 2006 "Fisica Astroparticellare: neutrini ed universo primordiale" by italian MIUR. S. Pastor was supported by the ILIAS project (contract No. RII3-CT-2004-506222), by the Spanish grants FPA2005-01269 (MEC) and ACOMP07-270 (Generalitat Valenciana), and by a Ramón y Cajal contract.

[1] L. Anchordoqui and F. Halzen, Annals Phys. 321, 2660 (2006) arXiv:hep-ph/0510389.

[2] V. A. Balkanov et al., Astropart. Phys. 12, 75 (1999) arXiv:astro-ph/9903341.

[3] J. Ahrens et al. [AMANDA Collaboration], Phys. Rev. D 66, 012005 (2002) arXiv:astro-ph/0205109].

[4] J. Ahrens et al. [IceCube Collaboration], Astropart. Phys. 20, 507 (2004) arXiv:astro-ph/0305196].

[5] M. Spurio [ANTARES Collaboration], arXiv:hep-ph/0611032

[6] G. Aggouras et al. [NESTOR Collaboration], Nucl. Instrum. Meth. A 567, 452 (2006).

[7] P. Piattelli [NEMO Collaboration], Nucl. Phys. Proc. Suppl. 165, 172 (2007).

[8] U. F. Katz, Nucl. Instrum. Meth. A 567, 457 (2006) arXiv:astro-ph/0606068.

[9] D. Hooper, Phys. Rev. D 65, 097303 (2002) arXiv:hep-ph/0203239.

[10] S. Hussain, D. Marfatia, D. W. McKay and D. Seckel, Phys. Rev. Lett. 97, 161101 (2006) arXiv:hep-ph/0606246.

[11] L. A. Anchordoqui, J. L. Feng and H. Goldberg, Phys. Rev. Lett. 96, 021101 (2006) arXiv:hep-ph/0504228.

[12] A. Cuoco et al., JCAP 0702, 007 (2007) arXiv:astro-ph/0609241. 
[13] S. Palomares-Ruiz, A. Irimia and T. J. Weiler, Phys. Rev. D 73, 083003 (2006) arXiv:astro-ph/0512231.

[14] J. Álvarez-Muñiz, F. Halzen, T. Han and D. Hooper, Phys. Rev. Lett. 88, 021301 (2002) arXiv:hep-ph/0107057.

[15] E. Waxman and J. N. Bahcall, Phys. Rev. D 59, 023002 (1999) arXiv:hep-ph/9807282.

[16] R. Gandhi, C. Quigg, M. H. Reno and I. Sarcevic, Phys. Rev. D 58, 093009 (1998) arXiv:hep-ph/9807264.

[17] J.G. Learned and S. Pakvasa, Astropart. Phys. 3, 267 (1995) arXiv:hep-ph/9405296.

[18] G. Miele, S. Pastor and O. Pisanti, Phys. Lett. B 634, 137 (2006) arXiv:astro-ph/0508038].

[19] J. Abraham et al. [Pierre Auger Collaboration], Nucl. Instrum. Meth. A 523, 50 (2004).

[20] National Geophysical Data Center, 2001, http://www.ngdc.noaa.gov/mgg/fliers/01mgg04.html

[21] R. Gandhi, C. Quigg, M. H. Reno and I. Sarcevic, Astropart. Phys. 5, 81 (1996) arXiv:hep-ph/9512364].

[22] J. F. Beacom, N. F. Bell, D. Hooper, S. Pakvasa and T. J. Weiler, Phys. Rev. D 68, 093005 (2003) [Erratum-ibid. D 72, 019901 (2005)] arXiv:hep-ph/0307025.

[23] F. Halzen and D. Hooper, Rept. Prog. Phys. 65, 1025 (2002) arXiv:astro-ph/0204527.

[24] C. Aramo et al., Astropart. Phys. 23, 65 (2005) arXiv:astro-ph/0407638.

[25] S. I. Dutta, Y. Huang and M. H. Reno, Phys. Rev. D 72, 013005 (2005) arXiv:hep-ph/0504208.

[26] J. F. Beacom et al., Phys. Rev. D 68, 093005 (2003); [Erratum-ibid. D 72, 019901 (2005)] arXiv:hep-ph/0307025].

P. D. Serpico and M. Kachelrieß, Phys. Rev. Lett. 94, 211102 (2005) arXiv:hep-ph/0502088.

T. Kashti and E. Waxman, Phys. Rev. Lett. 95, 181101 (2005) arXiv:astro-ph/0507599.

P. D. Serpico, Phys. Rev. D 73, 047301 (2006) arXiv:hep-ph/0511313.

Z. Z. Xing and S. Zhou, Phys. Rev. D 74, 013010 (2006) arXiv:astro-ph/0603781].

P. Lipari, M. Lusignoli and D. Meloni, Phys. Rev. D 75, 123005 (2007) arXiv:0704.0718 [astro-ph]].

M. Kachelrieß and R. Tomàs, Phys. Rev. D 74, 063009 (2006) arXiv:astro-ph/0606406.

M. Kachelrieß, S. Ostapchenko and R. Tomàs, arXiv:0708.3047 [astro-ph].

[27] Talk of E. de Wolf at RICAP07, June 20-22, 2007, Rome, Italy.

[28] S. Baker and R. D. Cousins, Nucl. Instrum. Meth. A 221, 437 (1984). 